\title{
Multidisciplinary approach for post-acute COVID-19 syndrome: time to break down the walls
}

\author{
David Montani $\mathbb{1}^{1,2,3}$, Laurent Savale $\mathbb{C}^{1,2,3}$, Antoine Beurnier ${ }^{1,2,4}$, Romain Colle $\mathbb{C}^{1,5}$, Nicolas Noël ${ }^{1,6}$, \\ Tài Pham (10) ${ }^{1,7}$, Xavier Monnet $\mathbb{( \mathbb { D }}^{1,2,7}$ and Marc Humbert (10)
}

${ }^{1}$ Université Paris-Saclay, Faculty of Medicine, Le Kremlin-Bicêtre, France. ${ }^{2}$ INSERM UMR_S 999, Hôpital Marie Lannelongue, Le Plessis Robinson, France. ${ }^{3}$ Dept of Respiratory and Intensive Care Medicine, AP-HP, Pulmonary Hypertension National Referral Centre, Hôpital Bicêtre, DMU 5 Thorinno, Le Kremlin-Bicêtre, France. ${ }^{4}$ Dept of Physiology - Pulmonary Function Testing, AP-HP, DMU 5 Thorinno, Hôpital Bicêtre, Le Kremlin-Bicêtre, France. ${ }^{5}$ Service de Psychiatrie, AP-HP, Hôpital de Bicêtre, DMU 11, équipe MOODS, INSERM U1018, CESP (Centre de Recherche en Epidémiologie et Santé des Populations), Le Kremlin-Bicêtre, France. ${ }^{6}$ Service de Médecine Interne et Immunologie Clinique, AP-HP, Hôpital de Bicêtre, DMU 7 Endocrinologie-Immunités-Inflammations-CancerUrgences, Le Kremlin-Bicêtre, France. ${ }^{7}$ Service de Médecine Intensive-Réanimation, AP-HP, Hôpital de Bicêtre, DMU 4 CORREVE Maladies du Coeur et des Vaisseaux, FHU Sepsis, Le Kremlin-Bicêtre, France.

Corresponding author: David Montani (david.montani@aphp.fr)

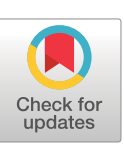

Copyright $\odot$ The authors 2021.

This version is distributed under the terms of the Creative Commons Attribution Non-Commercial Licence 4.0. For commercial reproduction rights and permissions contact permissions@ersnet.org

Received: 14 April 2021 Accepted: 19 April 2021

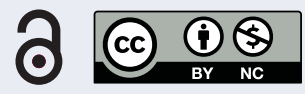

Shareable abstract (@ERSpublications)

The importance of the COVID-19 pandemic requires us to break down the walls between medical specialties to optimise the management of patients with post-acute COVID-19 syndrome https://bit. ly/3gzqEik

Cite this article as: Montani D, Savale L, Beurnier A, et al. Multidisciplinary approach for post-acute COVID-19 syndrome: time to break down the walls. Eur Respir J 2021; 58: 2101090 [DOI: 10.1183/ 13993003.01090-2021].
Severe acute respiratory syndrome coronavirus 2 (SARS-CoV-2) has provoked an ongoing global pandemic of coronavirus disease 2019 (COVID-19), which has affected 130 million people worldwide and caused nearly 3 million deaths in just over 1 year [1]. In 2020, COVID-19 represented the third highest cause of death in the USA after heart disease and cancer [2], and this outbreak has led to the largest drop in life expectancy since World War II [2]. Evidence-based medical treatment of COVID-19 (anticoagulants, corticosteroids, anti-inflammatory drugs, oxygenation therapy and ventilation) [3] seems to have improved patients' outcomes, and the vast majority will recover spontaneously or after acute phase management.

Several studies have reported the diversity of long-term complications of COVID-19 with a variety of symptoms and organ-related injuries, which has been referred to as "long COVID" or "post-acute COVID-19 syndrome" [4]. Even if the frequency of these complications is not high, the massive number of people who have been infected with SARS-CoV-2 suggests that this will represent a public health issue leading to a major consumption of healthcare resources. The issue of long COVID has been identified as a clear priority of the utmost importance for the World Health Organization [5].

The precise definition of these long-term complications remains to be defined, as well as the duration of chronic symptoms after initial presentation. The term "post-acute COVID-19 syndrome" was proposed to define symptoms and abnormalities persisting or present beyond 12 weeks of the onset of acute COVID-19 and not attributable to alternative diagnoses $[4,6]$. This term appears more appropriate than the unclear and worrying wording "long COVID”, which could falsely suggest the presence of a chronic SARS-CoV-2 infection. Nevertheless, the mechanisms leading to long-term complications of COVID-19 remain to be elucidated, although it is likely that they are diverse, encompassing a variety of local and systemic mechanisms. Besides potential sequelae of initial organ injuries, the questions of enhancing factors, such as persisting low grade inflammation, endothelial dysfunction or possible viral reservoirs, must be addressed [7, 8]. They may also include post-traumatic factors, especially in hospitalised patients, culminating in those treated in intensive care units. The impact of initial management and the SARS-CoV-2 variants on the type and incidence of long-term symptoms remains unresolved. 
Many long-term complications of COVID-19 have been reported to date (table 1). Of these, dyspnoea may be a direct consequence of lung infection with SARS-CoV-2, due to pulmonary sequelae detected by chest imaging (ground glass opacities, fibrotic lesions, sequelae of acute pulmonary embolism) and functional testing (restrictive pattern, low diffusing capacity of lung for carbon monoxide, persistent hypoxaemia). In addition to these direct sequelae of infection, other causes of respiratory symptoms may be observed, such as dysfunctional breathing or muscle weakness. However, the exploration of patients with post-acute COVID-19 syndrome requires a multidisciplinary approach, as complications far exceed the problem of dyspnoea, and it appears that pulmonary and systemic complications can be intimately entangled. Among the many complications are general signs (asthenia, diffuse pain), psychiatric symptoms (post-traumatic stress syndrome, depression, anxiety, insomnia), neurological symptoms (cognitive impairment, dysautonomia), and specific organ lesions affecting cardiovascular, renal, endocrine, ear-nose-throat, gastrointestinal or dermatological systems (table 1) [9-16]. Most patients with post-acute COVID-19 syndrome experience multiple and overlapping symptoms, in addition to a significant impact on their quality of life [10, 12-15]. Of note, such complications have been described in other post-acute settings and may not be specific to COVID-19 $[17,18]$. However, the emergence of these chronic symptoms requires a structured response to understand, treat and, most importantly, prevent it.

Given the diversity of symptoms, the management of these patients cannot be limited to a single specialised clinic and requires a multidisciplinary team approach. This is the experience we were able to carry out during the COMEBAC (Consultation MultiExpertise de Bicêtre Après COVID-19) uncontrolled cohort study, which included 478 adult patients discharged from Bicêtre Hospital (Université Paris-Saclay) [15]. This study strongly supports our vision that screening via telehealth consultation followed by a multidisciplinary assessment in outpatient clinics involving multiple specialists on the same day in a single location is likely the most effective way to manage the complexity of post-acute COVID-19 syndrome. This structured muldisciplinary team approach allows to offer a set of careful investigations necessary for each patient, with the aim of individualised medicine and the rationalisation of healthcare resources. To date, most studies have

\section{TABLE 1 Symptoms related to post-acute COVID-19 syndrome}

Organ systems

General assessment

Respiratory

Psychiatric and neurological
Post-acute COVID-19 symptoms

Asthenia

Muscle weakness

Diffuse pain

Myalgia, joint pain

Weight loss

Deterioration of quality of life

Dyspnoea

Cough

Radiologic sequelae

Functional impairment

Dysfunctional breathing

Chronic oxygen dependence

Post-traumatic stress

Depression

Anxiety

Insomnia

Headache

Cognitive impairment (brain fog)

Dysautonomia

\begin{tabular}{|c|c|}
\hline Cardiovascular & $\begin{array}{l}\text { Chest pain } \\
\text { Palpitations } \\
\text { Autonomic dysfunction } \\
\text { Myocardial fibrosis } \\
\text { Venous thromboembolic disease }\end{array}$ \\
\hline Renal & Persistent impaired renal function \\
\hline Ear-nose-throat & $\begin{array}{l}\text { Persistent anosmia or parosmia } \\
\text { Persistent ageusia }\end{array}$ \\
\hline Endocrine & $\begin{array}{l}\text { Thyroiditis } \\
\text { Onset or worsening of diabetes }\end{array}$ \\
\hline Dermatological & $\begin{array}{l}\text { Hair loss } \\
\text { Skin rash }\end{array}$ \\
\hline Gastrointestinal & Diarrhoea \\
\hline
\end{tabular}


focused on patients formerly hospitalised for COVID-19, but it is important to underscore that post-acute COVID-19 syndrome also exists in outpatients.

Post-acute COVID-19 syndrome clearly represents a global threat to mankind. A better understanding of the mechanisms, predisposing factors, and evolution (after 6 months) of these symptoms will require broad international cooperation, in order to offer efficacious preventative and curative approaches. The European Respiratory Network for Data-sharing in COVID-19 (END-COVID), initiated by the European Respiratory Society to merge different national initiatives in Europe studying the long-term effects of COVID-19, represents a unique opportunity to answer unresolved issues [19].

If we are to learn from the positive aspects of this dramatic episode, we will keep in mind the solidarity and adaptability of healthcare teams and the need to break down the walls between medical specialties to optimise the management of patients with post-acute COVID-19 syndrome.

Acknowledgement: We thank the patients, relatives, healthcare providers, administrative hospital teams and the writing committee of the COMEBAC study.

Conflict of interest: D. Montani reports grants and personal fees from Actelion and Bayer, grants, personal fees and non-financial support from MSD, personal fees from GSK, Pfizer, Chiesi, Boehringer and Incyte Biosciences France, non-financial support from Acceleron, outside the submitted work. L. Savale has nothing to disclose. A. Beurnier reports personal fees from Sanofi, outside the submitted work. R. Colle has nothing to disclose. N. Noël has nothing to disclose. T. Pham has nothing to disclose. X. Monnet has nothing to disclose. M. Humbert reports grants and personal fees from Actelion and Bayer, personal fees from Acceleron, GSK, Merck, Novartis, AstraZeneca and Sanofi, outside the submitted work.

\section{References}

1 World Health Organization. WHO Coronavirus (COVID-19) Dashboard. Date last accessed: 10 Apr 2021. https:// covid19.who.int

2 Ahmad FB, Anderson RN. The leading causes of death in the US for 2020. JAMA 2021; 325: 1829-1830.

3 Chalmers JD, Crichton ML, Goeminne PC, et al. Management of hospitalised adults with coronavirus disease-19 (COVID-19): a European Respiratory Society living guideline. Eur Respir J 2021; 57: 2100048.

4 Nalbandian A, Sehgal K, Gupta A, et al. Post-acute COVID-19 syndrome. Nat Med 2021; 27: 601-615.

5 Wise J. Long covid: WHO calls on countries to offer patients more rehabilitation. BMJ 2021; 372: n405.

6 COVID-19 Rapid Guideline: Managing the Long-Term Effects of COVID-19. London, National Institute for Health and Care Excellence (UK), 2020. Available from www.ncbi.nlm.nih.gov/books/NBK567261/

7 Ren X, Wen W, Fan X, et al. COVID-19 immune features revealed by a large-scale single-cell transcriptome atlas. Cell 2021; 184: 1895-1913.e19.

8 Kruglikov IL, Shah M, Scherer PE. Obesity and diabetes as comorbidities for COVID-19: underlying mechanisms and the role of viral-bacterial interactions. eLife 2020; 9: e61330.

9 Chopra V, Flanders SA, O'Malley M, et al. 60-day outcomes among patients hospitalized with COVID-19. Ann Intern Med 2021; 174: 576-578.

10 Carfi A, Bernabei R, Landi F, et al. Persistent symptoms in patients after acute COVID-19. JAMA 2020; 324: 603-605.

11 Carvalho-Schneider C, Laurent E, Lemaignen A, et al. Follow-up of adults with noncritical COVID-19 two months after symptom onset. Clin Microbiol Infect 2021; 27: 258-263.

12 Arnold DT, Hamilton FW, Milne A, et al. Patient outcomes after hospitalisation with COVID-19 and implications for follow-up: results from a prospective UK cohort. Thorax 2020; 76: 399-401.

13 Halpin SJ, Mclvor C, Whyatt G, et al. Postdischarge symptoms and rehabilitation needs in survivors of COVID-19 infection: a cross-sectional evaluation. J Med Virol 2021; 93: 1013-1022.

14 Huang C, Huang L, Wang Y, et al. 6-month consequences of COVID-19 in patients discharged from hospital: a cohort study. Lancet 2021; 397: 220-232.

15 Writing Committee for the COMEBAC Study Group, Morin L, Savale L, et al. Four-month clinical status of a cohort of patients after hospitalisation for COVID-19. JAMA 2021; 325: 1525-1534. .

16 Bellan M, Soddu D, Balbo PE, et al. Respiratory and psychophysical sequelae among patients with COVID-19 four months after hospital discharge. JAMA Netw Open 2021; 4: e2036142.

17 Masclans JR, Roca O, Muñoz X, et al. Quality of life, pulmonary function, and tomographic scan abnormalities after ARDS. Chest 2011; 139: 1340-1346.

18 Pandharipande PP, Girard TD, Jackson JC, et al. Long-term cognitive impairment after critical illness. N Engl J Med 2013; 369: 1306-1316.

19 European Respiratory Society. END-COVID - European Respiratory Network for Data-Sharing in COVID19. Date last accessed: 05 Apr 2021. www.ersnet.org/science-and-research/clinical-research-collaboration-applicationprogramme/end-covid-european-respiratory-network-for-data-sharing-in-covid19/ 